\title{
A SHORT PROOF OF A GREENE THEOREM
}

\author{
CHUNG-LIE WANG ${ }^{1}$
}

\begin{abstract}
A short and simple proof of an inequality of the Gronwall type is given for a class of integral systems based upon the generalized Gronwall lemma of Sansone-Conti.
\end{abstract}

Recently David E. Greene [1] used a technically involved iteration in proving the following

TheOREM [GREene]. Let $K_{1}, K_{2}$ and $\mu$ be nonnegative constants and let $f, g$ and $h_{i}$ be continuous nonnegative functions for all $t \geqslant 0$ with $h_{i}$ bounded such that

$$
\begin{aligned}
& f(t) \leqslant K_{1}+\int_{0}^{t} h_{1}(s) f(s) d s+\int_{0}^{t} e^{\mu s} h_{2}(s) g(s) d s, \\
& g(t) \leqslant K_{2}+\int_{0}^{t} e^{-\mu s} h_{3}(s) f(s) d s+\int_{0}^{t} h_{4}(s) g(s) d s
\end{aligned}
$$

for all $t \geqslant 0$. Then there exist constants $c_{i}$ and $M_{i}$ such that

$$
f(t) \leqslant M_{1} e^{c_{1} t}, \quad g(t) \leqslant M_{2} e^{c_{2} t}
$$

for all $t \geqslant 0$.

In this note, is presented a short and simple proof of this theorem based upon the generalized Gronwall lemma of Sansone-Conti which is cited from [2] in a restricted form as follows

(Generalized) Gronwall Lemma. For all $t \geqslant 0$, let three functions $\lambda, \phi, u$ be given such that $\lambda$ is summable and nonnegative, $\phi$ is absolutely continuous, and $u$ is continuous. If $u(t) \leqslant \phi(t)+\int_{0}^{t} \lambda(s) u(s) d s$, then

$$
u(t) \leqslant \int_{0}^{t} \phi^{\prime}(s) \exp \left(\int_{s}^{t} \lambda(r) d r\right) d s+\phi(0) \exp \left(\int_{0}^{t} \lambda(r) d r\right) .
$$

PROOF OF THE THEOREM. Let $P$ be an upper bound for $h_{i}$ (the assumption $\mu>0$ is not necessarily required here), then

$$
\begin{aligned}
& f(t) \leqslant K_{1}+P \int_{0}^{t} f(s) d s+P \int_{0}^{t} e^{\mu s} g(s) d s \\
& g(t) \leqslant K_{2}+P \int_{0}^{t} e^{-\mu s} f(s) d s+P \int_{0}^{t} g(s) d s .
\end{aligned}
$$

Received by the editors July 5, 1977.

AMS (MOS) subject classifications (1970). Primary 34C10; Secondary 45F05.

${ }^{1}$ The author was supported (in part) by the N.R.C. of Canada (Grant No. A3116). 
Multiplying (1) by $e^{-\mu t}$ and then adding to (2),

$$
\begin{aligned}
& e^{-\mu} f(t)+g(t) \\
& \leqslant K_{1} e^{-\mu t}+K_{2}+\int_{0}^{t} P\left[e^{\mu(s-t)}+1\right]\left[e^{-\mu s} f(s)+g(s)\right] d s \\
& \leqslant K_{1} e^{-\mu t}+K_{2}+\int_{0}^{t} 2 P\left[e^{-\mu s} f(s)+g(s)\right] d s .
\end{aligned}
$$

Applying the lemma to (3)

$$
\begin{aligned}
e^{-\mu t} f(t) & +g(t) \\
& \leqslant \int_{0}^{t}\left(-K_{1} \mu e^{-\mu s}\right) \exp \left(\int_{s}^{t} 2 P d r\right) d s+\left(K_{1}+K_{2}\right) \exp \left(\int_{0}^{t} 2 P d s\right) \\
& =\frac{K_{1} \mu}{2 P+\mu} e^{-\mu t}+\frac{2 P\left(K_{1}+K_{2}\right)+K_{2} \mu}{2 P+\mu} e^{2 P t} .
\end{aligned}
$$

The conclusion of the theorem is now clear.

\section{REFERENCES}

1. David E. Greene, An inequality for a class of integral systems, Proc. Amer. Math. Soc. 62 (1977), 101-104.

2. G. Sansone and R. Conti, Non-linear differential equations, rev. ed., Internat. Ser. of Monographs in Pure and Appl. Math., Vol. 67, Macmillan, New York, 1964. MR 31 \# 1417.

Department of Mathematics, University of Regina, Regina, Saskatchewan, Canada S4S $\mathrm{OA} 2$ 